\title{
Introduction to the Minitrack on Digital Methods
}

\author{
Shawn Walker \\ Arizona State University \\ shawn.w@asu.edu
}

\author{
Alexander Halavais \\ Arizona State University \\ theprof@asu.edu
}

\author{
Matthew Weber \\ University of Minnesota \\ msw@umn.edu
}

\section{Introduction}

The Digital Methods minitrack focuses on addressing the methodological challenges of conducting research with digital and social media data. This is a new minitrack at HICSS, and was conceived as a forum to advance methodological discussions and to highlight innovations in digital research. While there has been an explosion of research using social media data to study human and machine behavior, the accompanying digital methods literature continues to lag. Researchers, practitioners, and students are left to adapt existing, "non-digital" methods or to reinvent methods via a process of trial and error. The papers presented in this minitrack highlight new approaches to digital methodology, and aim to detail specific methodological advances that can benefit the research community at large.

\section{Advancing digital methods in research}

There has been an explosion of research using social media data to study human behavior and social interaction in almost every domain of social science. While the body of literature using digital and social media data is growing at a staggering rate, accompanying methodological contributions about the process of conducting research with digital and social media data remains thin. The existing methodological literature is typically tool or technology driven, and not a result of empirical examination of the data collection process. This leaves researchers without an understanding of how to approach or evaluate the social media data collection process, and in turn, how to appropriately interpret findings from this type of research.

This minitrack addresses this gap by providing a venue to discuss methodological issues and approaches to conducting research with digital and social media data. We welcome papers related to methodological challenges for researchers including, but not limited to: (1) the need for new methods for data collection and analysis, (2) adaptations of existing methods (3) issues of representation and sampling, (4) ephemerality of social media data, (5) holistic collection of digital social media data and associated content such as images/URLs/video, (6) preservation, archiving, and data sharing, and (7) impact of changing platform affordances, interfaces, designs, and APIs.

The two papers in the minitrack this year address the themes above and advance our understanding of the platform algorithms that shape the posts users and researchers receive. The following sections will provide more detailed explanations of each paper.

\section{Researching Algorithms and Recommendation Systems Inside Out Using Reversed Engineering}

The research developed by Pettersen details a "reversed engineering" approach to examining algorithms and the effects of algorithms. The case study used in the paper examines an online dating platform to better understand how users of the platforms are paired with potential matches. The reversed engineering approach focuses the researcher's attention on the black box of an algorithm by tracing behaviour through "observable leaks, openings, and shortcomings." In the case study, ghost profiles are created and used to trace the behavior of the algorithm by tracing and recording observations. The work gives scholars a useful framework for studying a wide range of algorithmic behavior on digital media, including the distribution of news media, the recommendation of products and the provision of "friend' recommendations. 


\section{Profiling Online Social Network Platforms: Twitter vs. Instagram}

The second paper in this minitrack builds on the theme of better understanding the behavior of algorithms and online social networks. The paper by Ayora, Horita and Kamienski, investigates Twitter and Instagram to understand how the platforms' message characteristics impact key variables of data completeness and latency. The focus is on understanding how messages sent by one user are received by another. Completeness captures the number of messages sent by one user that are seen by another; latency captures any delay in receiving those messages. The authors outline an approach that involves recording the environment configuration, developing an experimental design testing the publishing of key messages, then posting and capturing those messages to capture information about the completeness of the message posted and any latency. The model provides another useful approach for better understanding of the way in which these frequently used digital platforms disseminate and communicate messages to users.

\section{Future Directions for Digital Methods}

This year the Digital Methods minitrack provided a forum for starting new conversations about the development of methods that engage specifically with the challenges of conducting research in a digital media environment. In recent years, the increasing focus on a need to better understand human and machine behavior in online environments is increasingly critical to our knowledge of the world around us. We look forward to developing these conversations in future years as this minitrack continues. 